\title{
Model recovery anti-windup compensator design for magnitude and rate saturated LPV systems
}

\author{
T. Péni, Z. Szabó, B. Vanek, J. Bokor
}

\begin{abstract}
This paper extends the LTI anti-windup compensator scheme proposed in [6] to linear parameter-varying (LPV) systems. Following the MRAW concept, the dynamical part of the compensator is formed by the exact copy of the plant. The design procedure is thus simplified to the construction of a parameter-dependent state feedback, which stabilizes the plant's copy and determines the performance and the domain of applicability of the compensator. To decrease the conservatism, the presented method applies parameter-dependent Lyapunov function and embeds the saturation (dead-zone) in a parameterdependent sector. The design is formulated as an LMI-based convex optimization problem.

The paper also investigates the possibility of eliminating certain free variables in order to reduce the complexity of the synthesis procedure. It is shown that an elimination procedure similar to that in [6] can be carried out, but with LPV systems the reconstruction of the compensator gain is not so straightforward. To overcome the difficulty a novel method is proposed, which is based on a closed formula parameterizing all solutions of the synthesis LMIs. Both of the fully parameterized and the reduced complexity syntheses are presented and their properties are analyzed. The applicability of the methods is demonstrated on a simple LPV plant.
\end{abstract}

\section{INTRODUCTION}

Control input limitations are always present in real physical systems. If the controller is designed irrespective of these limitations, the later appearance of a saturation may cause undesired behavior in the closed loop: it leads to performance degradation or even instability. This effect is called controller windup.

One possible way to minimize the undesired effects of controller windup is using an anti-windup compensator. The concept is simple ([17], [21]): the controller is designed irrespective of the saturation and then a a static or dynamic compensator is designed so that the following three criteria are fulfilled: (1) the closed loop is (locally) stable; (2) if there is no saturation, the nominal performance is guaranteed; (3) in case of saturation the system is driven by the compensator so that the signals leave the saturating domain and the nominal performance is recovered as quickly as possible.

There are two large groups of model-based anti-windup solutions ([21], [8]): Direct Linear Anti-Windup (DLAW) and Model Recovery Anti-Windup (MRAW) methods. The direct method considers the unconstrained feedback loop as a special plant and the compensator as a special controller. The compensator construction is then reformulated as a control design problem for which the existing synthesis algorithms - LTI [12] or LPV [2] - can be easily adapted. Since the plant in the compensator design consists of the original plant and the controller, the LMIs are formulated in the extended (plant+controller dimensional) space. If the plant is a high dimensional system and the unconstrained controller inherits this complexity, the optimization problem may become large and computationally demanding. On the other hand, the DLAW concept has a significant advantage over the other approach: as it is based on the robust control machinery, the modeling errors and uncertainties can be easily integrated into the design procedure [2].

This paper focuses on the other class, the model-recovery (MRAW) solutions. In the MRAW framework the dynamics

The authors are with the Systems and Control Laboratory of Computer and Automation Research Institute of HAS, Budapest, 1518, P.O.B. 63., Hungary. Corresponding author: T. Peni, pt@scl.sztaki.hu of the compensator is the exact copy of the plant and the compensation is structured so that the difference between the real plant and its copy carries all information about the ideal (saturation-free) behavior [21] [6], [17]. If there is no uncertainty the MRAW compensator is totally independent of the actual controller, so it can work even with a nonlinear controller. Moreover, the computational complexity of MRAW algorithms is generally low, because the dynamics of the compensator are fixed and thus only a state feedback gain has to be designed. Though all advantages of MRAW algorithms can be exploited only if the plant is precisely known, in particular cases the methods can be extended to uncertain systems as well [16], [10].

Most papers addressing the anti-windup problem consider only LTI plants. Since the LPV structure is a powerful extensions of the LTI class, it makes sense to investigate the possibilities of extending the LTI anti-windup solutions to parameter varying models. In case of DLAW design the extension is a bit easier because the parameter-dependence can be easily integrated in the general control design framework ([19], [13], [2], [20]). Although the problem is more delicate in the MRAW framework, some approaches have already been extended to parameter-varying plants. For example, in [11] the anti-windup compensator structure presented in [17] is extended to LPV systems. This method is based on the coprime-factors of the plant, which is a conceptually different approach than that is applied in this paper. Moreover, the algorithm proposed in [11] is derived only for magnitude saturation. The authors of [6] have already proposed in [3] one possible LPV extension of the same compensator design concept we also use in this paper. In contrast to our approach, [3] handles only magnitude saturation and the compensator is based on the polytopic controlled invariant set of the plant Due to the approach the authors chose, the method of [3] requires the LPV plant to be embedded in a polytopic model

In this paper the LTI-MRAW compensator scheme described in [4] and [6] is extended to LPV plants. The proposed method remains applicable if both magnitude and rate saturation are present and applies quadratic Lyapunov function with ellipsoidal level sets; both can be easily computed even for large dimensional plants. To decrease the conservatism, the Lyapunov function is chosen to be parameterdependent and the saturation (dead-zone) is embedded in a parameter-dependent sector. The design is formulated as an LMI-based convex optimization problem. The paper also investigates the possibility of eliminating certain free variables in order to reduce the complexity of the design procedure. It is shown that an elimination procedure is similar to that in [5], but the reconstruction of the compensator gain is not so straightforward. To overcome the difficulty a novel method is proposed, which is based on a closed formula parameterizing all solutions of the synthesis LMIs. Both of the fully parameterized and the reduced complexity syntheses are presented and their properties are analyzed. The applicability of the methods is demonstrated on a simple LPV plant.

The paper is organized as follows. After the introduction and the definitions of notations the anti-windup compensator design is presented for LPV systems in Section III. The problems related to the variable elimination are discussed in Section IV. Section V is devoted to the numerical example. The paper is concluded in Section VI. 


\section{NOTATION}

The notations used in the paper are fairly standard. If $x$ is a vector then its $i$-th component is denoted by $x_{i}$. The $i$-th column of a matrix is indicated by $[X]_{i}$. The saturation $\left(\operatorname{sat}_{R}(u)\right)$ and dead-zone $\left(\mathrm{dz}_{R}(u)\right)$ functions are defined in the standard way: $\operatorname{sat}_{R}(u)_{i}=\max \left(\min \left(u_{i}, R_{i}\right),-R_{i}\right)$ and $\mathrm{dz}_{R}(u)=u-\operatorname{sat}_{R}(u)$. A set is polyhedral if it is defined as an intersection of finite number of closed halfspaces. The polytope is a bounded polyhedral set. Polytopes and polyhedral sets can be defined by their hyperplane representation: $\mathcal{P}(H, h)=\{x: H x \leq h\}$, where $\leq$ is defined element-wise. A 0-symmetric polyhedral set is defined by $\mathcal{P}(H)=\left\{x:|H x|_{\infty} \leq 1\right\}$, where $|\cdot|_{\infty}$ denotes a matrix norm. For simplicity, the matrix sum $M+M^{T}$ is denoted by $H e(M)$.

\section{MRAW COMPENSATOR DESIGN FOR LPV SYSTEMS}

In this section the LTI anti-windup compensator design method proposed by Forni et. al in [6] is extended to linear parameter varying systems. For this, we start from an LPV plant given in the standard state-space form:

$$
\begin{aligned}
\dot{x} & =A(\rho) x+B_{u}(\rho) u+B_{d}(\rho) d \\
y & =C_{y}(\rho) x+D_{y u}(\rho) u+D_{y d}(\rho) d \\
z & =C_{z}(\rho) x+D_{z u}(\rho) u+D_{z d}(\rho) d
\end{aligned}
$$

where $x \in \mathbb{R}^{n}, u \in \mathbb{R}^{m}$ and $y \in \mathbb{R}^{p}, z \in \mathbb{R}^{n_{z}}$ are the state, the control input, the measured- and the performance output, respectively. The external inputs $r$ and $d$ are the reference and the disturbance. The external, time varying parameter scheduling the dynamics is denoted by $\rho$. Let $\rho \in \mathbb{R}^{p}$ and let $\dot{\rho}$ be denoted by $\nu$. We assume that $\rho$ is available for measurement and the upper and lower bounds of each $\rho_{i}$ and $\nu_{i}$ are a-priori known: $\rho_{i} \leq \rho_{i} \leq \bar{\rho}_{i}$, $\underline{\nu}_{i} \leq \nu_{i} \leq \bar{\nu}_{i}, \forall i=1, \ldots, p$. Since the aim of the antiwindup compensation is to improve the performance of an existing controller, we assume that a nominal (scheduled) controller has already been designed for the plant:

$$
\begin{aligned}
& \dot{x}_{c}=f\left(x_{c}, \rho, u_{c}, r\right) \\
& y_{c}=g\left(x_{c}, \rho, u_{c}, r\right)
\end{aligned}
$$

The general form indicates that the controller can be of arbitrary structure, even nonlinear. If there is no saturation the nominal interconnection defined by the relation $u=y_{c}$, $u_{c}=y$ is assumed to provide the intended behavior: it is globally stable and meets all performance specifications prescribed for output $z$. (To distinguish the nominal system from the saturated one the trajectory of signals generated by the nominal closed loop will be denoted by $\hat{\imath}$, i.e. $\hat{x}, \hat{z}$, etc.)

The saturated system is defined by inserting the magnitude and rate limited actuator, defined as follows, between the controller output and plant input:

$$
\dot{u}=\operatorname{diag}(R) \operatorname{sign}\left(\operatorname{sat}_{M}(v)-u\right) \doteq \operatorname{sat}_{M R}(v), \quad v=y_{c}
$$

where $M, R$ are vectors, containing the magnitude and rate saturation limits: $M=\left[M 1, \ldots, M_{m}\right], M=\left[R 1, \ldots, R_{m}\right]$. Dynamics (3) is one possible model that is able to mimic the the actuator's behavior. The advantages of this particular structure are detailed e.g. in [7].

Since our anti-windup compensator follows the MRAW

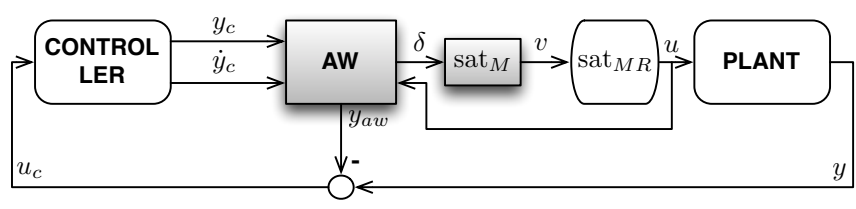

Fig. 1. The AW compensation scheme

concept ([21]), it is based on the model of the plant:

$$
\begin{aligned}
\dot{x}_{a w} & =A(\rho) x_{a w}+B_{u}(\rho)\left(u-y_{c}\right) \\
\dot{\delta} & =\operatorname{sat}_{R}\left(\dot{y}_{c}+v_{1}\right) \\
y_{a w} & =C_{y}(\rho) x_{a w}+D_{y u}(\rho)\left(u-y_{c}\right) \\
z_{a w} & =C_{z}(\rho) x_{a w}+D_{z u}(\rho)\left(u-y_{c}\right) \\
v & =\operatorname{sat}_{M}(\delta), v_{1}=k\left(\rho,\left[\begin{array}{c}
x_{a w} \\
\delta-y_{c}
\end{array}\right]\right)
\end{aligned}
$$

In LTI case $\dot{y}_{c}$ can be directly computed from the controller's state if the controller is strictly proper. In LPV case, the properness is not enough for precise computation, because the derivative generally depends also on $\dot{\rho}$. In most cases only an acceptable approximation is available by using e.g. the proper filter $F(s)=\frac{s}{1+\tau s}, \tau \ll 1$. (On the correct choice of $\tau$, e.g. [5] can provide further information.) In (4) $k_{1}$ feedback is a free component, which will be determined later on. The compensated closed loop consisting of (1)-(2)-(3)(4) is defined via the following interconnection (Fig. 1.):

$$
u=\operatorname{sat}_{M R}(v), \quad u_{c}=y-y_{a w}
$$

The reason why the particular structure (4) is stressed becomes clear if one computes the difference between the states of the compensator and the plant:

$$
\begin{aligned}
\dot{x}-\dot{x}_{a w} & =A(\rho)\left(x-x_{a w}\right)+B_{u}(\rho) y_{c}+B_{d}(\rho) d \\
y-y_{a w} & =C_{y}(\rho)\left(x-x_{a w}\right)+D_{y d}(\rho) d \\
z-z_{a w} & =C_{z}(\rho)\left(x-x_{a w}\right)+D_{z u}(\rho) y_{c}+D_{z d}(\rho) d \\
\dot{x}_{c} & =f\left(x_{c}, \rho, y-y_{a w}, r\right) \\
y_{c} & =g\left(x_{c}, \rho, y-y_{a w}, r\right)
\end{aligned}
$$

It is easy to see that (6) describes the same dynamics as the nominal closed loop, so in case of $\left(x-x_{a w}\right)(0)=x(0)$, (i.e. $\left.x_{a w}(0)=0\right)$ the trajectory of (6) coincides with the trajectory of the nominal closed loop system, i.e. $y_{c}=\hat{u}=$ $\hat{y}_{c},\left(x-x_{a w}\right)(t)=\hat{x}(t),\left(z-z_{a w}\right)(t)=\hat{z}(t)$.

If the actuator saturates and consequently the state of the system starts to diverge from the ideal behavior, the state difference will still evolve along the desired, unsaturated trajectory. Exploiting this property, the saturation effect can be compensated for and the ideal behavior can be recovered if $z$ is forced to converge to the ideal output $z-z_{a w}$, i.e. to make $z_{a w} \rightarrow 0$. This is the goal, which has to be achieved by suitably choosing the conditioning signal $v_{1}$.

To design $v_{1}$ we start from the dynamics of the AWcompensator

$$
\begin{aligned}
\dot{x}_{a w} & =A(\rho) x_{a w}+B_{u}(\rho)\left(u-y_{c}\right) \\
& =A(\rho) x_{a w}+B_{u}(\rho) \operatorname{sat}_{M \epsilon}\left(\delta_{a w}\right)+B_{u}(\rho) \sigma_{M} \\
\dot{\delta}_{a w} & =\dot{\delta}-\dot{y}_{c}=\operatorname{sat}_{R}\left(\dot{y}_{c}+v_{1}\right)-\dot{y}_{c} \\
& =\operatorname{sat}_{R \epsilon}\left(v_{1}\right)+\sigma_{R}
\end{aligned}
$$

where the final formulas are obtained by applying the following lemma proven in [6]:

Lemma 1. Given any pair $v, y \in \mathbb{R}$ and $\varepsilon \in(0,1)$, there exists $\epsilon \in[\varepsilon, 2-\varepsilon]$ s.t. the following equality holds:

$$
\operatorname{sat}_{S}(y+v)-y=\operatorname{sat}_{S \epsilon}(v)+\sigma_{S}
$$

where $\left|\sigma_{S}\right| \leq\left|2 \mathrm{dz}_{S(1-\varepsilon)}(y)\right|$. 
In our case, $\left|\sigma_{M}\right| \leq\left|2 \mathrm{dz}_{M(1-\varepsilon)}\left(y_{c}\right)\right|=\left|2 \mathrm{dz}_{M(1-\varepsilon)}(\hat{u})\right|$, $\left|\sigma_{R}\right| \leq 2 \mathrm{dz}_{R(1-\varepsilon)}\left(\dot{y}_{c}\right)|=| 2 \mathrm{dz}_{R(1-\varepsilon)}(\dot{\hat{u}}) \mid$, that is the "external", disturbance-like signals $\sigma_{M}, \sigma_{R}$ are proportional to the difference between the saturated and the unsaturated (ideal) control inputs, so they measure the effect of saturation.

If the compensator feedback is chosen to be linear, but parameter-dependent, i.e. $k(\cdot)=K(\rho)\left[\begin{array}{l}x_{a w} \\ \delta_{a w}\end{array}\right]=$ $\left[K_{x}(\rho) K_{\delta}(\rho)\right]\left[\begin{array}{l}x_{a w} \\ \delta_{a w}\end{array}\right]$ then we get equations (8), which are similar to those obtained in the LTI case. In (8), $S_{i}=M_{i}$, $S_{m+i}=R_{i}, i=1 \ldots m$ and

$$
\begin{aligned}
& q_{\epsilon} \doteq \mathrm{dz}_{S \varepsilon}\left(\left(\begin{array}{cc}
0 & I_{m} \\
K_{x}(\rho) & K_{\delta}(\rho)
\end{array}\right)\left[\begin{array}{l}
x_{a w} \\
\delta_{a w}
\end{array}\right]\right) \\
& =\left[\mathrm{dz}_{M \epsilon}^{T}\left(\delta_{a w}\right) \mathrm{dz}_{R \epsilon}^{T}\left(K_{x} x_{a w}+K_{\delta} \delta_{a w}\right)\right]^{T}
\end{aligned}
$$

Following the concept of [6], we assume that the actuators spend only finite time in saturating mode, i.e. $\sigma_{M}$ and $\sigma_{R}$ have compact support $[0, T]$. The effect of saturation can be described as short-term disturbance (realized by $\sigma_{M}$ and $\sigma_{R}$ ), which pushes the state of (8) from the origin to some nonzero value $\left(x_{a w}(T), \delta_{a w}(T)\right)$ in $T$ seconds. Then, by assumption, $\sigma_{M}$ and $\sigma_{R}$ drop back to zero. Clearly, if $\left(x_{a w}(T), \delta_{a w}(T)\right)$ is inside the domain of attraction of (8), (where the domain of attraction is defined at $\sigma_{M}=\sigma_{R}=0$ ), then the states $\left(x_{a w}, \delta_{a w}\right)$ will return to zero, i.e. the closed loop dynamics returns to the intended behavior.

It is now clear, if we maximize - via the state feedback gain $K(\rho)$ - the domain of attraction of (8), then we can improve the applicability of the AW compensator: the larger the domain of attraction is, the longer and larger the "disturbance" signals $\left(\sigma_{R}, \sigma_{M}\right)$ the system tolerates.

The most straightforward way to characterize the domain of attraction of (8) is to consider the level set of the Lyapunov function:

$$
\mathcal{E}(P(\rho), 1)=\left\{\left[\begin{array}{l}
x_{a w} \\
\delta_{a w}
\end{array}\right] \mid V\left(\rho,\left[\begin{array}{l}
x_{a w} \\
\delta_{a w}
\end{array}\right]\right) \leq 1\right\}
$$

In this paper the Lyapunov function is chosen to be parameter-dependent and quadratic:

$$
\begin{gathered}
V\left(\rho,\left[\begin{array}{l}
x_{a w} \\
\delta_{a w}
\end{array}\right]\right)=\left[\begin{array}{l}
x_{a w} \\
\delta_{a w}
\end{array}\right]^{T} P(\rho)\left[\begin{array}{l}
x_{a w} \\
\delta_{a w}
\end{array}\right], \\
P(\rho) \in \mathbb{R}^{n+m} P(\rho)>0, \forall \rho
\end{gathered}
$$

The stability is guaranteed if $\dot{V}<-2 \alpha V$ for all $q_{\epsilon}$ with some $\alpha>0$. As long as the state is inside the parameterdependent polyhedral set $\mathcal{H}(\rho):\left|(\varepsilon S)^{-1} H(\rho)\left[\begin{array}{l}x_{a w} \\ \delta_{a w}\end{array}\right]\right|_{\infty} \leq$ $1, q_{\epsilon}$ satisfies the modified sector condition [9]:

$$
\begin{aligned}
& q_{\epsilon}^{T} U\left(q_{\epsilon}-\left[\begin{array}{cc}
0 & I_{m} \\
K_{x}(\rho) & K_{\delta}(\rho)
\end{array}\right]\left[\begin{array}{l}
x_{a w} \\
\delta_{a w}
\end{array}\right]\right. \\
& \left.+H(\rho)\left[\begin{array}{l}
x_{a w} \\
\delta_{a w}
\end{array}\right]\right) \leq 0 \quad \forall\left[\begin{array}{l}
x_{a w} \\
\delta_{a w}
\end{array}\right] \in \mathcal{H}(\rho)
\end{aligned}
$$

Note that, in LPV case it is possible to choose parameterdependent polyhedral sets to follow the variation of the underlying system. By applying the S-procedure this condition can be added to $\dot{V}<-2 \alpha V$, which yields the following sufficient condition of stability:

$$
\begin{array}{r}
\dot{V}(\rho)-2 q_{\varepsilon}^{T} U\left(q_{\varepsilon}-\left[\begin{array}{cc}
0 & I_{m} \\
K_{x}(\rho) & K_{\delta}(\rho)
\end{array}\right]\left[\begin{array}{l}
x_{a w} \\
\delta_{a w}
\end{array}\right]\right. \\
\left.+H(\rho)\left[\begin{array}{l}
x_{a w} \\
\delta_{a w}
\end{array}\right]\right) \leq-2 \alpha V\left(\rho,\left[\begin{array}{l}
x_{a w} \\
\delta_{a w}
\end{array}\right]\right)
\end{array}
$$

Performing the time derivation and substituting the dynamics of the compensator we obtain the following matrix inequality:

$$
\left[\begin{array}{c}
x_{a w} \\
\delta_{a w} \\
q_{\varepsilon}
\end{array}\right]^{T} \Omega\left[\begin{array}{c}
x_{a w} \\
\delta_{a w} \\
q_{\varepsilon}
\end{array}\right] \leq 0
$$

where $\Omega$ is given by (12). Multiplying $\Omega$ from left and right by $\left[\begin{array}{cc}P(\rho)^{-1} & 0 \\ 0 & U^{-1}\end{array}\right]=\left[\begin{array}{cc}Q(\rho) & 0 \\ 0 & \operatorname{diag}\left(W_{M}, W_{R}\right)\end{array}\right]$ the terms $H(\rho) Q(\rho)$ and $\frac{1}{2} \sum_{i=1}^{p} Q(\rho) \frac{\partial P(\rho)}{\partial \rho_{i}} Q(\rho) \nu_{i}$ appear. The first term can be replaced by a new variable $X(\rho)=H(\rho) Q(\rho) \in$ $\mathbb{R}^{2 m \times(m+n)}$, while the equality

$$
\frac{\partial Q(\rho)}{\partial \rho_{i}}=-Q(\rho) \frac{\partial P(\rho)}{\partial \rho_{i}} Q(\rho)
$$

can be applied to the second. With $\partial Q(\rho, \nu)=$ $-\frac{1}{2} \sum_{i=1}^{p} \frac{\partial Q(\rho)}{\partial \rho_{i}} \nu_{i}$ we can rewrite (11) to (13), where $L(\rho)=$ $\left[K_{x}(\rho) K_{\delta}(\rho)\right] Q(\rho) \in \mathbb{R}^{m \times m+n}$. Note that, inequality (13) is linear in $L(\rho), Q(\rho) X(\rho)$ and $\nu$, but it is not linear in $\rho$. In order to convert the infinite number of constraints in (13) into a numerically tractable form, the structure of the parameter dependent variables has to be fixed. One possible choice is the affine form: $Q(\rho)=Q_{0}+\sum_{i=1}^{p} \rho_{i} Q_{i}$, $X(\rho)=X_{0}+\sum_{i=1}^{p} \rho_{i} X_{i}, L(\rho)=L_{0}+\sum_{i=1}^{p} \rho_{i} L_{i}$. The next step is defining a suitably dense grid over the domain of $\rho$, which together with the corner points of the domain of $\nu$ gives the following finite set of parameter values:

$$
\begin{array}{r}
\mathfrak{G}=\left\{\left(\rho_{1}^{\left(k_{1}\right)}, \ldots, \rho_{p}^{\left(k_{p}\right)}, \nu_{1}^{\left(l_{1}\right)}, \ldots, \nu_{p}^{\left(l_{p}\right)}\right) \mid\right. \\
\left.\rho_{i}^{\left(k_{i}\right)}=\underline{\rho}_{i}+k_{i} \frac{\bar{\rho}_{i}-\underline{\rho}_{i}}{N_{i}}, k_{i} \in\left\{1, \ldots, N_{i}\right\}, \nu_{i}^{\left(l_{i}\right)} \in\left\{\underline{\nu}_{i}, \bar{\nu}_{i}\right\}\right\}
\end{array}
$$

The number of grid points is $2^{p} \prod_{i} N_{i}$. Evaluated at each grid point, inequality (13) generates a finite set of linear matrix inequalities, which have to be simultaneously solved for variables $Q_{i}, X_{i}, L_{i}, W_{M}, W_{R}$.

The next group of synthesis inequalities comes from the containment relation $\mathcal{E}(P(\rho), 1) \subset \mathcal{H}(\rho)$, which can be expressed in the following way [1]:

$$
0 \leq\left[\begin{array}{cc}
\varepsilon^{2} S_{i}^{2} Q(\rho) & {[X(\rho)]_{i}^{T}} \\
{[X(\rho)]_{i}} & 1
\end{array}\right], \quad i=1, \ldots, 2 m
$$

To maximize the parameter-dependent level-sets a ball of radius $\beta$ is placed inside $\mathcal{E}(P(\rho), 1)$ and $\beta$ is maximized. If $\mathcal{B}=\left\{x \mid x^{T} x \leq \beta\right\}$ then $\mathcal{B} \subset \mathcal{E}(P(\rho), 1)$ holds if

$$
\beta I \leq Q(\rho)
$$

In practical applications it is worth limiting the compensator input $v_{1}$ in order to avoid large jumps in the control signal. Prescribing a direct gain limit $K(\rho)^{T} K(\rho) \leq k_{\max }^{2} I$ ([6]) cannot be applied in LPV case because it leads to nonlinear constraints in the free variables. Instead, the norm of $v_{1}$ is limited over $\mathcal{E}(P(\rho), 1)$ by involving an additional LMI condition [1]:

$$
\left[\begin{array}{ll}
v_{\max }^{2} I & L(\rho) \\
L(\rho)^{T} & Q(\rho)
\end{array}\right]>0
$$

Summarizing the results, we get a similar optimization problem as in LTI case:

$$
\max _{Q_{i}, X_{i}, L_{i}, W_{M}, W_{R}} \beta
$$

subject to (13), (14), (15), (16), for all $(\rho, \nu) \in \mathfrak{G}$

From the obtained parameters the compensator gain can be computed as $K(\rho)=L(\rho) Q(\rho)^{-1}$.

\section{ELIMINATION OF VARIABLES}

In this section an approach is proposed for decreasing the complexity of the design procedure (17). The method is based on eliminating unknowns $L(\rho)$ and $W_{R}$ from the 


$$
\begin{aligned}
& {\left[\begin{array}{c}
\dot{x}_{a w} \\
\dot{\delta}_{a w}
\end{array}\right]=\left(\begin{array}{cc}
A(\rho) & B_{u}(\rho) \\
K_{x}(\rho) & K_{\delta}(\rho)
\end{array}\right)\left[\begin{array}{l}
x_{a w} \\
\delta_{a w}
\end{array}\right]-\left(\begin{array}{cc}
B_{u}(\rho) & 0 \\
0 & I
\end{array}\right) q_{\epsilon}+\left(\begin{array}{cc}
B_{u}(\rho) & 0 \\
0 & I
\end{array}\right)\left[\begin{array}{c}
\sigma_{M} \\
\sigma_{R}
\end{array}\right]}
\end{aligned}
$$

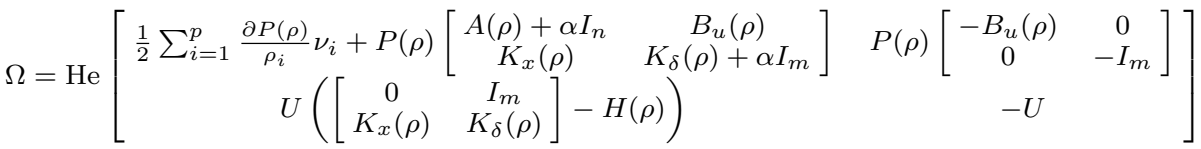

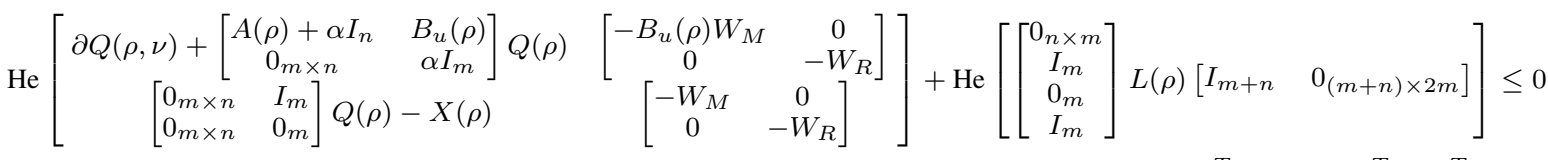

synthesis inequalities. To derive the modified procedure we prove first the following lemma:

Lemma 2. Consider the following matrix inequality

$$
\left(\begin{array}{ccc}
P_{11} & P_{12}+X^{T} & P_{13} \\
P_{12}^{T}+X & P_{22} & 0 \\
P_{13}^{T} & 0 & P_{33}
\end{array}\right)<0
$$

in free variable $X$. This inequality has an unstructured solution $X$ if and only if

$$
\left(\begin{array}{cc}
P_{11} & P_{13} \\
P_{13}^{T} & P_{33}
\end{array}\right)<0 \text { and } \quad\left(\begin{array}{cc}
P_{22} & 0 \\
0 & P_{33}
\end{array}\right)<0
$$

Moreover, if the conditions above hold then all solutions of (18) can be expressed in closed form as follows:

$$
X=-P_{12}^{T}+\left(-P_{22}\right)^{1 / 2} \mathbf{K}\left(P_{13} P_{33}^{-1} P_{13}^{T}-P_{11}\right)^{1 / 2}
$$

where $\mathbf{K}$ is an arbitrary contraction matrix, i.e. $\mathbf{K}^{T} \mathbf{K}<I$.

Proof. The proof of the solvability conditions (19) can be found e.g. in [14] or can be easily checked as follows. The necessity of (19) is trivial: it is enough to cancel the first or second block row/column of (18). For sufficiency, note that $P_{33}<0$ (consequence of the second inequality of (19)) and thus (18) is equivalent to the following inequality (Schur complement)

$$
\left(\begin{array}{cc}
P_{11} & P_{12}+X^{T} \\
P_{12}^{T}+X & P_{22}
\end{array}\right)-\left(\begin{array}{c}
P_{13} \\
0
\end{array}\right) P_{33}^{-1}\left(\begin{array}{ll}
P_{13}^{T} & 0
\end{array}\right)<0
$$

If (19) holds then the diagonal blocks are negative. Any $X$ rendering the off-diagonal block zero (e.g. $X=-P_{12}^{T}$ ) is a suitable solution of (18).

Now we can prove formula (20). For this, (21) is rewritten first as follows

$$
\left(\begin{array}{cc}
P_{11}-P_{13} P_{33}^{-1} P_{13}^{T} & P_{12}+X^{T} \\
P_{12}^{T}+X & P_{22}
\end{array}\right)<0
$$

Since $P_{22}<0$ (second inequality of (19)) the Schur theorem can be applied again. The inequality above is thus equivalent to

$$
\begin{array}{r}
P_{11}-P_{13} P_{33}^{-1} P_{13}^{T}-\left(P_{12}+X^{T}\right) P_{22}^{-1}\left(P_{12}^{T}+X\right)= \\
=\Pi-P_{12} P_{22}^{-1} P_{12}^{T}-P_{12} P_{22}^{-1} X-X^{T} P_{22}^{-1} P_{12}^{T}-X^{T} P_{22}^{-1} X \\
=\left(\begin{array}{c}
I \\
X
\end{array}\right)^{T}\left(\begin{array}{cc}
\Pi-P_{12} P_{22}^{-1} P_{12}^{T} & -P_{12} P_{22}^{-1} \\
-P_{22}^{-1} P_{12}^{T} & -P_{22}^{-1}
\end{array}\right)\left(\begin{array}{c}
I \\
X
\end{array}\right)<0
\end{array}
$$

where $\Pi=P_{11}-P_{13} P_{33}^{-1} P_{13}^{T}$. The solvability conditions imply that $\Pi<0$. So, we can perform the following factorization:

$$
\left(\begin{array}{cc}
\Pi-P_{12} P_{22}^{-1} P_{12}^{T} & -P_{12} P_{22}^{-1} \\
-P_{22}^{-1} P_{12}^{T} & -P_{22}^{-1}
\end{array}\right)=M^{T}\left(\begin{array}{cc}
-I & 0 \\
0 & I
\end{array}\right) M
$$

where

$$
M=\left(\begin{array}{cc}
(-\Pi)^{1 / 2} & 0 \\
\left(-P_{22}\right)^{-1 / 2} P_{12}^{T} & \left(-P_{22}\right)^{-1 / 2}
\end{array}\right)
$$

By applying the matrix inverse lemma the inverse of $M$ can be expressed as follows:

$$
\begin{aligned}
& M^{-1}=\left(\begin{array}{cc}
(-\Pi)^{-1 / 2} & 0 \\
-\left(-P_{22}\right)^{1 / 2}\left(-P_{22}\right)^{-1 / 2} P_{12}^{T}(-\Pi)^{-1 / 2} & \left(-P_{22}\right)^{1 / 2}
\end{array}\right) \\
& =\left(\begin{array}{cc}
(-\Pi)^{-1 / 2} & 0 \\
-P_{12}^{T}(-\Pi)^{-1 / 2} & \left(-P_{22}\right)^{1 / 2}
\end{array}\right)=\left(\begin{array}{ll}
N_{11} & N_{12} \\
N_{21} & N_{22}
\end{array}\right)
\end{aligned}
$$

From $M^{-1}$ all solutions of (18) can be generated by Möbius transformation [15]:

$$
\begin{aligned}
X & =\left(N_{21}+N_{22} \mathbf{K}\right)\left(N_{11}+N_{12} \mathbf{K}\right)^{-1} \\
& =-P_{12}^{T}+\left(-P_{22}\right)^{1 / 2} \mathbf{K}(-\Pi)^{1 / 2}
\end{aligned}
$$

where $\mathbf{K}$ is an arbitrary contraction.

Remark 1. The solvability conditions (19) of Lemma 2. have already been proved in several papers and books (e.g. [14]). The novelty in Lemma 2. is formula (20) describing all solutions of the LMI.

Remark 2. Note, the solution $X$ is linear in $\mathbf{K}$. This makes it possible to use $\mathbf{K}$ as a decision variable in a linear matrix inequality.

To apply Lemma 2. to the synthesis inequalities, (13) has to be transformed first to the required form. For this, consider the following nonsingular, linear transformation $S=$ $\left(\begin{array}{llll}S_{1} & S_{2} & S_{3} & S_{4}\end{array}\right)$ with $S_{3}=Y_{\perp} \bigcap Z_{\perp},\left(\begin{array}{ll}S_{1} & S_{3}\end{array}\right)=Y_{\perp}$, $\left(\begin{array}{ll}S_{2} & S_{3}\end{array}\right)=Z_{\perp}$, where $Y_{\perp}$ and $Z_{\perp}$ denote the orthogonal complements of $Y$ and $Z$, respectively. In our case $S$ takes the following form:

$$
S=\left(\begin{array}{lll}
S_{1} & S_{2} & S_{3}
\end{array}\right)=\left[\begin{array}{cc|c|c}
I_{n} & 0 & 0 & 0 \\
0 & I_{m} & 0 & 0 \\
0 & 0 & 0 & I_{m} \\
0 & -I_{m} & I_{m} & 0
\end{array}\right], S_{4}=\emptyset
$$

Then, instead of (13), we can consider the transformed inequality $S^{T}(13) S<0$ :

$(Y S)^{T} L(\rho)(Z S)+(Z S)^{T} L(\rho) Y S+S^{T} \Phi(\rho, \nu) S<0$

By using (23), (22) can be rewritten as follows:

$$
\begin{aligned}
& \operatorname{He}\left(\frac{\Phi_{11}(\rho, \nu)}{\frac{\Phi_{21}(\rho, \nu)-\Phi_{41}(\rho)}{\Phi_{41}(\rho)+L_{11}(\rho)}}\right. \\
& \left.\begin{array}{c|c|c}
\Phi_{12}(\rho, \nu) & 0 & -B_{u} W_{M} \\
\Phi_{22}(\rho, \nu)-\Phi_{42}(\rho) & 0 & 0 \\
\hline \Phi_{42}(\rho)+W_{R}+L_{22}(\rho) & -W_{R} & 0 \\
\hline \Phi_{32}(\rho) & 0 & -W_{M}
\end{array}\right)<0
\end{aligned}
$$

Now we can apply Lemma 2 . to the set of linear matrix inequalities generated by (24) at grid points $(\rho, \nu) \in \mathfrak{G}$. In 


$$
\begin{aligned}
(Y S)^{T} L(\rho)(Z S) & =\left[\begin{array}{c}
0_{n \times m} \\
0_{m} \\
I_{m} \\
0_{m}
\end{array}\right] L(\rho)\left[\begin{array}{cccc|c|c}
I_{n} & 0 & 0 & 0 \\
0 & I_{m} & 0 & 0
\end{array}\right]=\left(\begin{array}{ccc|c}
0_{n} & 0 & 0 & 0 \\
0 & 0_{m} & 0 & 0 \\
\hline L_{11}(\rho) & L_{12}(\rho) & 0_{m} & 0 \\
\hline 0 & 0 & 0 & 0_{m}
\end{array}\right) \\
S^{T} \Phi(\rho, \nu) S & =\operatorname{He}\left(\begin{array}{cc|c|c}
\Phi_{21}(\rho, \nu)-\Phi_{41}(\rho) & \Phi_{22}(\rho, \nu)-\Phi_{42}(\rho) & 0 & -B_{u} W_{M} \\
\hline \Phi_{41}(\rho) & \Phi_{42}(\rho)+W_{R} & -W_{R} & 0 \\
\hline \Phi_{31}(\rho) & \Phi_{32}(\rho) & 0 & -W_{M}
\end{array}\right)
\end{aligned}
$$

our case the solvability conditions amount to the following inequalities:

$$
\begin{aligned}
\operatorname{He}\left(\begin{array}{cc|c}
\Phi_{11}(\rho, \nu) & \Phi_{12}(\rho, \nu) & -B_{u} W_{M} \\
\Phi_{21}(\rho, \nu)-\Phi_{41}(\rho) & \Phi_{22}(\rho, \nu)-\Phi_{42}(\rho) & 0 \\
\hline \Phi_{31}(\rho) & \Phi_{32}(\rho) & -W_{M}
\end{array}\right)<0 \\
\text { for all }(\rho, \nu) \in \mathfrak{G} \text { and } \operatorname{He}\left(\begin{array}{c|c}
-W_{R} & 0 \\
\hline 0 & -W_{M}
\end{array}\right)<0
\end{aligned}
$$

The second inequality always holds by definition, while the first is equivalent to $(25)$ Thus, $W_{R}$ and $L(\rho)$ have been successfully eliminated. The synthesis procedure will consist of two steps. In the first step, the domain of attraction $(\beta)$ is maximized subject to the solvability condition (25) (and (14), (15)), then, in a second step, the formula (20) is applied to reconstruct and shape the compensator gain $K$. More formally, the following optimization problem has to be solved first:

$$
\max _{Q_{i}, X_{i}, W_{M}} \beta
$$

subject to (25), (14), (15) for all $(\rho, \nu) \in \mathfrak{G}$

By applying formula (20) the missing variable $L$ (containing the compensator gain) can be expressed by using the obtained $Q, X, W_{M}$ matrices: the formula can be seen under reference (26). The free parameters in (26) are only the contraction $\mathbf{K}$ and the positive definite diagonal matrix $W_{R}$; they will be used to shape the feedback gain in a second step. For this, define the following LMI feasibility problem:

$$
\begin{aligned}
& \text { Find } \overline{\mathbf{K}}, W_{R} \text { s.t. } \\
& {\left[\begin{array}{cc}
v_{\max }^{2} I & L_{a}+\sqrt{2} \overline{\mathbf{K}} L_{b} \\
L_{a}^{T}+L_{b}^{T} \overline{\mathbf{K}}^{T} \sqrt{2} & Q(\rho)
\end{array}\right]>0, \forall(\rho, \nu) \in \mathfrak{G},(} \\
& \text { and }\left[\begin{array}{cc}
W_{R} & \overline{\mathbf{K}} \\
\overline{\mathbf{K}}^{T} & I
\end{array}\right]>0,(27 \mathrm{~b})
\end{aligned}
$$

The first inequality limits $v_{1}$ inside $\mathcal{E}(P(\rho), 1)$ and is obtained by introducing $\overline{\mathbf{K}}=W_{R}^{1 / 2} \mathbf{K}$ and substituting (26) into (16). The second inequality is equivalent to $\mathbf{K}^{T} \mathbf{K}<I$, which prescribes for $\mathbf{K}$ to be a contraction. (Note that, the number of free variables in (27a) and (27b) can be further increased if $\overline{\mathbf{K}}$ is defined in parameter-dependent, - e.g. affine - form.) Having determined $\mathbf{K}$ and $W_{R}$ the parameter-dependent feedback gain can be computed as follows: $K(\rho, \nu)=L(\rho, \nu) Q(\rho)^{-1}$.

Remark 3. Note that, the solution $L$ above depends on both $\rho$ and $\nu$. This is not surprising if one compares this result with other LPV (control-) design methods, see e.g. [18], where the controller reconstructed from the solution of the reduced synthesis LMIs depends on the time derivative of the scheduling parameter as well. If $\dot{\rho}$ is difficult to measure or estimate one can choose $\mathbf{K} \equiv 0$, since $L_{a}$ does not depend on $\nu$. An other, rather practical solution could be the following: after solving (25), all or some of the variables $Q_{i}, X_{i}$ and $W_{M}$ can be fixed and then the original inequality (13) can be re-solved for $K$ and $W_{R}$. In this case the parameterdependence of $K$ has to be a-priori fixed (e.g. in affine form). Since (25) does not guarantee that (13) has a solution in the fixed, specific structure, therefore this practical feedback construction procedure may fail.
Remark 4. If one compares our approach to other methods, (see e.g. [18] or [14]) one significant difference can be found: although all approaches eliminate the controller variable from the synthesis inequalities, the methods in the cited papers construct then only one, particular controller from the solution of the reduced LMIs. In our case there is a parameterized set of possible feedback gains from which the designer can select the "best one" via optimization ((27)).

Remark 5. By applying variable elimination we might expect larger domain of attraction, because in this case the structure of $L$ and $K$ is not a-priori fixed.

\section{NUMERICAL EXAMPLE}

The AW design procedure and the applicability of the parameter-dependent compensator are demonstrated on a simple LPV plant:

$$
\begin{aligned}
& A(\rho)=\left[\begin{array}{cc}
-0.5 & 1 \\
0.8 & -0.4
\end{array}\right]+\rho\left[\begin{array}{cc}
-0.4 & 1 \\
0.9 & -0.4
\end{array}\right] \\
& B_{u}(\rho)=\left[\begin{array}{c}
-0.2 \\
-5
\end{array}\right]+\rho\left[\begin{array}{c}
-0.6 \\
2
\end{array}\right], C_{z}=\left[\begin{array}{ll}
1 & 0
\end{array}\right], \quad D_{z u}=0
\end{aligned}
$$

We assume $-0.2 \leq \rho \leq 0.8,-5 \leq \nu \leq 5$ and both states are available for measurement. The aim of the control is to track a given reference signal with output $z$. For this, the following servo controller has been designed:

$$
y_{c}=K_{c}(\rho)\left[\begin{array}{l}
x_{1} \\
x_{2} \\
e_{I}
\end{array}\right], \quad e_{I}=\int r-z d t
$$

Between the controller output and plant input there is an actuator with magnitude and rate limits: $M=1$ and $R=10$. The scheduling parameter is varying according to Figure 2. The nominal and the saturated closed loop responses are depicted in Figure 3. It can be seen, the saturation results in the loss of stability, so the design of anti-windup compensation makes sense. At first the design with the full parameter set (Section III) was performed. This was followed by the 2-step procedure using reduced complexity LMIs (Section IV). In both cases the decay rate was $\alpha=2$. We obtained $\beta_{\text {full }}=0.0369$ with the first and $\beta_{\text {elim }}=0.0372$ with the second algorithm. The closed loop responses are plotted also in Figure 3. (The time derivative of the controller output $\left(\dot{y}_{c}\right)$ was computed approximately by using the filter $s /(0.01 s+1))$. It can be seen, that both algorithms gave acceptable solutions, but the compensator generated by the 2 -step procedure provided better performance. The reason of the better transients is that the structure of the compensator gain was not fixed a-priori. (In the 2-step procedure the contraction $\mathbf{K}$ was determined via optimization (27) so the compensator gain depended also on $\nu$ ). The difference between the fully parameterized and the reduced complexity synthesis is more straightforward if one decrease $\alpha$ to 0.01 e.g. to increase the domain of attraction. In this case the fully parameterized design fails with numerical problems, while the reduced complexity synthesis still provides a feasible solution. (We obtained $\beta=3.0320$, which indicates significantly larger domain of attraction.)

\section{CONCLUSION}

In this paper the LTI anti-windup compensator design presented in [6] has been extended to parameter-varying plants. Two synthesis method have been derived: in the first case 


$$
\begin{array}{r}
\mathrm{He}\left(\begin{array}{rr}
\partial Q(\rho, \nu)+\left(\begin{array}{cc}
A(\rho)+\alpha I_{n} & B_{u}(\rho) \\
0 & \alpha I_{m}
\end{array}\right) Q(\rho)-\left(\begin{array}{cc}
0 & 0 \\
X_{21}(\rho) & X_{22}(\rho)
\end{array}\right) & -B_{u} W_{M} \\
0 \\
{\left[\begin{array}{ll}
0 & I_{m}
\end{array}\right] Q(\rho)-\left[X_{11}(\rho) X_{12}(\rho)\right]} & -W_{M}
\end{array}\right)<0 \text { for all }(\rho, \nu) \in \mathfrak{G} \\
L(\rho, \nu)=-\left[\begin{array}{ll}
\Phi_{41}(\rho) & \left.\Phi_{42}(\rho)+W_{R}\right]+\sqrt{2} W_{R}^{1 / 2} \mathbf{K} \cdot\left(\left[\begin{array}{cc}
\Phi_{31}(\rho)^{T}-B_{u} W_{M} \\
\Phi_{32}(\rho)^{T}
\end{array}\right]\left(-2 W_{M}\right)^{-1}\left[\begin{array}{c}
\Phi_{31}(\rho)^{T}-B_{u} W_{M} \\
\Phi_{32}(\rho)^{T}
\end{array}\right]^{T}\right. \\
\left.-H e\left[\begin{array}{cc}
\Phi_{11}(\rho, \nu) & \Phi_{12}(\rho, \nu) \\
\Phi_{21}(\rho, \nu)-\Phi_{41}(\rho) & \Phi_{22}(\rho, \nu)-\Phi_{42}(\rho)
\end{array}\right]\right)^{1 / 2}=L_{a}+\sqrt{2} W_{R}^{1 / 2} \mathbf{K} L_{b}
\end{array}\right.
\end{array}
$$

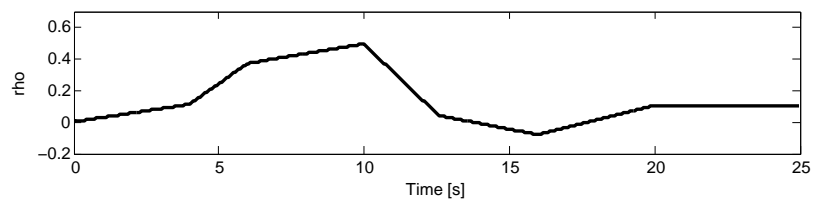

Fig. 2. Scheduling parameter $\rho$
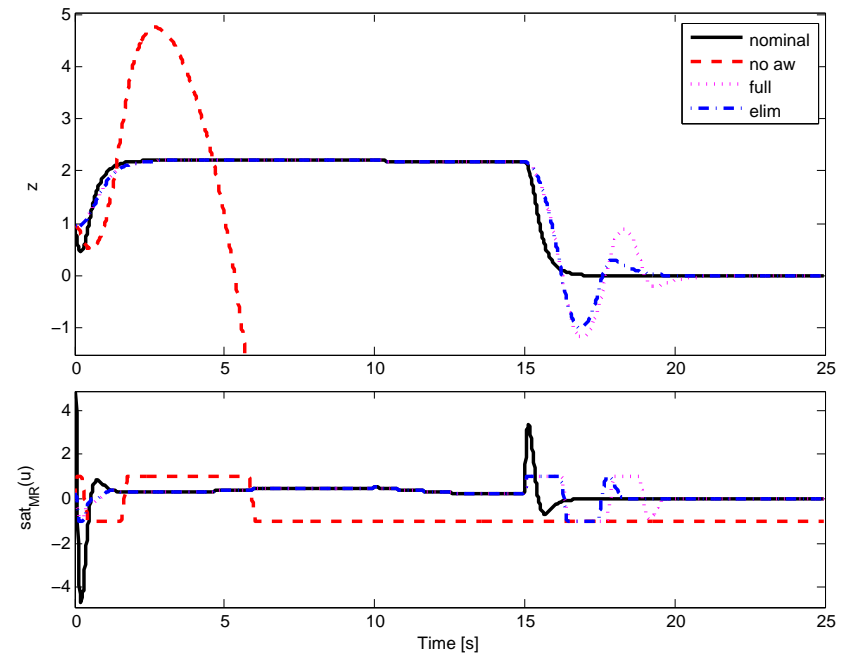

Fig. 3. Simulation results with $\alpha=2$ : output (top) and control input (bottom). 4 simulations are plotted: nominal closed loop (black), the effect of saturation (red), compensator of Section III (magenta), compensator of Section IV (blue).
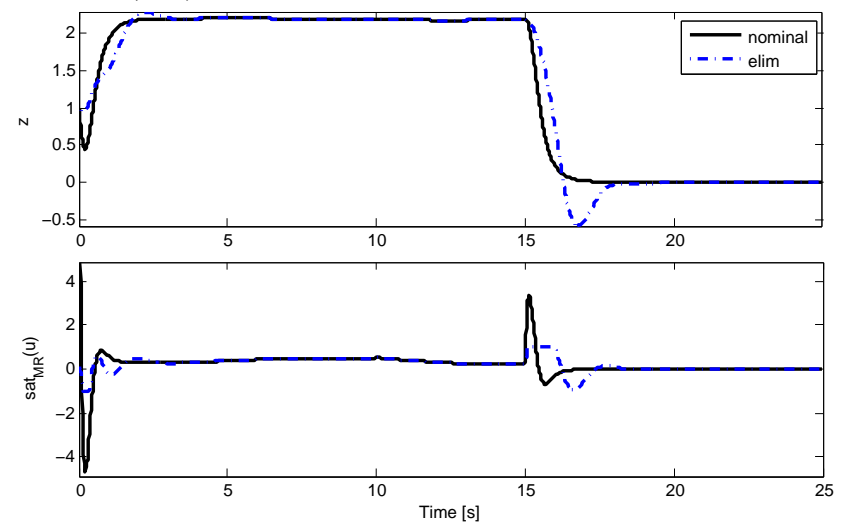

Fig. 4. Simulation results in case of $\alpha=0.01$ : output (top) and control input (bottom). Nominal closed loop (black) and compensator of Section IV (blue). (The fully parameterized design (Section III) has failed.)

more free variables are involved and the structure of the compensator is fixed. In the second algorithm the compensator gain is eliminated from the synthesis; only the Lyapunov function and the domain of attraction is determined first. The compensator gain is computed in a second step, from the parameterized set of all solutions of the synthesis LMIs. It was shown that this second approach has notable advantages over the first method: it is less conservative, as the parameter dependence of the feedback gain is not fixed a-priori and it is computationally more attractive, because it involves less free variables. The approaches are compared on a simple LPV plant.

\section{ACKNOWLEDGMENT}

The research leading to these results has received funding from the European Union Seventh Framework Programme (FP7/2007- 2013) under grant agreement n 2314544. Reconfiguration of Control in Flight for Integral Global Upset Recovery (Reconfigure) project, Andres Marcos coordinator.

\section{REFERENCES}

[1] S. Boyd, L. El Ghaoui, E. Feron, and V. Balakrishnan. Linear Matrix Inequalities in System and Control Theory, volume 15 of Studies in Applied Mathematics. Society for Industrial and Applied Mathematics (SIAM), 1994.

[2] G. Ferreres and J.-M. Biannic. Convex design of a robust antiwindup controller convex design of a robust antiwindup controller for an LFT model. IEEE Transactions on Automatic Control, 52(11):2173-2177, 2007.

[3] F. Forni and S. Galeani. Gain-scheduled, model-based anti-windup for LPV systems. Automatica, 46:222-225, 2010.

[4] F. Forni, S. Galeani, and L. Zaccarian. Model recovery anti-windup for plants with rate and magnitude saturation. In Eurpean Control Conference (ECC), pages 324-329, 2009

[5] F. Forni, S. Galeani, and L. Zaccarian. An almost anti-windup scheme for plants with magnitude, rate and curvature saturation. In American Control Conference, pages 6769-6774, 2010.

[6] F. Forni, S. Galeani, and L. Zaccarian. Model recovery anti-windup for continuous-time rate and magnitude saturated linear plants. Automatica, 48:1502-1513, 2012.

[7] S. Galeani, S. Onori, A.R.Teel, and L. Zaccarian. A magnitude and rate saturation model and its use in the solution of a static anti-windup problem. Systems \& Control Letters, 57:1-9, 2008.

[8] S. Galeani, S. Tarbouriech, M. Turner, and L. Zaccarian. A tutorial on modern anti-windup design. In European Control Conference (ECC), pages 306-323, 2009.

[9] J. M. Gomes and S. Tarbouriech. Antiwindup design with guaranteed regions of stability: An lmi-based approach. IEEE Transaction on Automatic Control, 50:106-111, 2005.

[10] A. Marcos, M. C. Turner, and I. Postlethwaite. An architecture for design and analysis of high-performance robust antiwindup compensators. IEEE Transactions on Automatic Control, 52(9):1672-1679, 2007.

[11] E. Prempain, M. C. Turner, and I. Postlethwaite. Coprime factor based anti-windup synthesis for parameter-dependent systems. Systems \& Control Letters, 58:810-817, 2009.

[12] C. Roos and J.-M. Biannic. A convex characterization of dynamicallyconstrained anti-windup controllers. Automatica, 44:2449-2452, 2008.

[13] C. Roos, J.-M. Biannic, S. Tarbouriech, C. Prieur, and M. Jeanneau. On-ground aircraft control design using a on-ground aircraft control design using a parameter-varying anti-windup approach. Aerospace design using a parameter-varying anti-windup
Science and Technology, 14(7):459-471, 2010.

[14] C. Scherer and S. Weiland. Lecture Notes DISC Course on Linear Matrix Inequalities in Control. 1999.

[15] Z. Szabó, Zs. Bíró, and J. Bokor. All controllers for an LPV robust control problem. In 7th IFAC Symposium on Robust Control Design (ROCOND), pages 337-342, 2012.

[16] M. C. Turner, G. Herrmann, and I. Postlethwaite. Accounting for uncertainty in anti-windup synthesis. In American Control Conference $(A C C)$, pages 5292-5297, 2004.

[17] P. F. Weston and I. Postlethwaite. Linear conditioning for systems containing saturating actuators. Automatica, 36:1347-1354, 2000.

[18] Fen Wu. Control of Linear Parameter Varying Systems. PhD thesis, University of California at Berkeley, 1995.

[19] Fen Wu, K. M. Grigoriadis, and A. Packard. Anti-windup controller design using linear parameter-varying control methods. International Journal of Control, 73(12):1104-1114, 2000.

[20] Fen Wu and M. Soto. Extended anti-windup control schemes for LTI and LFT systems with actuator saturations. International Journal of Robust and Nonlinear Control, 14(1255-1281), 2004.

[21] L. Zaccarian and A. R. Teel. Modern Anti-windup Synthesis. Princeton University Press, 2011. 